Aus dem serologischen Laboratorium der Staatskrankenanstalt und psychiatrischen Universitätsklinik Friedrichsberg in Hamburg.

(Direktor: Prof: Dr. Weygand t.)

\title{
Bemerkungen zu der Arbeit von Schönfeld „Über Befunde in der Rückenmarksflüssigkeit bei nerrengesunden Mensehen"
}

in $\mathrm{Nr} .5$ und 6 dieser Zeitschrift.

Von

\section{Privatdozent Dr. V. Kafka,}

Leiter des Laboratoriums.

Vor einiger Zeit haben Herrenschneider und Herrenschneider-Gumprich über positive Ergebnisse der Phase I in den Rückenmarksflüssigkeiten an nicht-organischen Nervenleiden Erkrankter berichtet. Diese Befunde hat Hauptmann einer Kritik unterzogen, die vollkommen den Erfahrungen entspricht, die wir im Laufe der Jahre an unserem großen Material gemacht haben.

Nun hat Schönfeld über Untersuchungsresultate berichtet, die in den Sätzen gipfeln, daß vollkommen Gesunde, bzw. solche, die an Krankheiten ohne Beteiligung des Nervensystems leiden, bei wiederholten Untersuchungen der Ruickenmarksflüssigkeit regelmäßig eine positive Phase I aufweisen, und zwar in $8 \%$ der Punktionen, daß ferner die $\mathrm{Pandysche}$ Reaktion in $42 \%$ der Punktionen und $59 \%$ der Fälle, eine positive Weichbrodtsche Reaktion in $58 \%$ der Punktionen und $68 \%$ der Fälle, schließlich die Emanuelsche Mastixreaktion bis zu $36 \%$ bzw. $10 \%$ bei Liquornormalen positiv auftreten können.

Es ist ohne weiteres klar, daß die oben erwähnten Reaktionen, bevor sie der Öffentlichkeit übergeben worden sind - und auch nachher - an einem großen Material von Fällen mit normalem Liquor erprobt worden sind, und daß. eben das negative Verhalten bei diesen Fällen erst die Grundlage für die diagnostische Verwendung unter pathologischen Verhältnissen abgegeben hat. Schönfelds überraschende Ergebnisse bedürfen daher der Kritik, da sie, wenn sie für die sicher 
normalen Rückenmarksflüssigkeitẹn verallgemeinert werden -würden, unsere heutigen liquordiagnostischen Richtlinien verschieben würden.

Fragen wir uns daher zuvörderst, welches Material Schönfeld seinen Untersuchungen zugrunde gelegt hat? Es besteht aus Gonorrhöen und Dermatosen ${ }^{1}$ ), ,,also jenen Fälle, die man im allgemeinen wohl als liquornormal betrachten darf. Daran ändern nichts einzelne in der Literatur niedergelegte abweichende Befunde":

Schon aus diesen Worten geht hervor, daß män ein solches Material keinesfalls ohne weiteres als liquornormal bezeichnèn darf.

Tch kann an dieser stelle nicht ausfuhrlicher auf die Literatur eingehen, sondern möchte nur an die Befunde Ferrands (Pleozytosë bei Dermatosen), die Yoanitescus and Galanescus (1906) and Henkels" (Pleozytose bei Gonorrhöe), sowie. C. Sterns (Phase I bei Gonorriöe), welch letztere auch Schönfeld erwähnt, erinnern: Wir wissen eben heute, daß einerseits Hautkrankheiten (wegen der Beziehungen $z u$ den Meningen), andererseits Infektionskrankheiten zu. wenn auch geringfügigen Affektionen der Meningen hinneigen. Das ist für alle die nichts Neues, die die Liquorliteratur seit 1904 verfolgt haben. Ein solches Material ist eben nicht gesund zu nennen; es ist zwar nervennormal, wenn man so will, da klinische Erscheinungen von seiten des Z.N.S. nicht bestehen, braucht aber nicht „meningennormal" zu sein. Wählt man aber solche Fälle als Ausgangspuñkte, um daraus Schlüsse auf wirklich liquornormale Fälle zu ziehen, so ist ein solches. Vorgehen nicht nur logisch unstatthaft.

Im einzelnen sei noch auf folgendes hingewiesen. Bezüglich der Phase I haben schon Nonne und seine Schüler angegeben, daß Spur Opaleszenz noch als negativ zu deuten ist, da sie durch die normalerweise vorhandenen Globuline hervorgerufen sein kann. Meist ist Spur Opaleszenz von deutlicher Opaleszenz, die wir nur bei organischen Nervenleiden und meningealen Affektionen sehen, genügend zu trennen. Es bestehen aber immerhín Fälle; wo die Differenzierung schwieriger und subjektivem Urteil unterworfen ist. Es darf aber auf keinen Fall Spur Opaleszenz als positiv gebucht werden, wie es z. B. Herrenschneider und Herrenschneider-Gumprich getan haben.

Die Pandysche Reaktion ist durch ihre hohe Empfindlichkeit

1) Im Speziellen 80 Fälle von Gonorrhöe, 4 von Ulcus molle, 18 von Hauttuberkulose, 20 von verschiedenen Hauterkrankungen, 1 von seniler Demenz, 2 von spitzen Kondylomen und 1 von Balanitis. Wo bleiben die ,Gesunden"? 
bekannt. Daher hat Zaloziecki seine Modifikation angegeben. Nur unter ihrer Zuhilfenahme und bei Kontrolleinstellung eines sicheren normalen Liquors darf daher heute ein Schluß aus einer PandyReaktion gezogen werden $\mathbf{1})$. Bezüglich der Weichbrodtschen Reaktion ist eine Arbeit aus unserem Laboratorium im Gange, auf die hier verwiesen sei. Was nun die Mastix reaktion betrifft, so ist nicht verwunderlich, wenn Schönfeld bei Anstellung der Reaktion nach Emanuel auch in wirklich normalen Fällen positive Ausschläge bekommen hat. Darauf haben Jacobsthal und Kafka und andere Autoren hingewiesen, da die 11/4\% ige Kochsalzlösung eine Elektrolytenkonzentration bedeutet, bei der viele nicht austitrierte Mastixversuchslösungen auch schon durch die normalen Liquoreiweißkörper ausgeflockt werden können. Benutzt man aber die Versuchsanordnung von Jacobsthal und Kafka, so kommen positive Reaktionen (Ausflockungen) bei wirklich normalem Liquor nicht vor; dafiir sprechen viele Hunderte von Reaktionen mit normalem Liquor. Die $10 \%$ positive Reaktionen, die Schönfeld, ,nach Anstellung eines Vorversuches" erhalten hat, sind für uns ein Zeichen, daß der Liquor pathologisch ist, vorausgesetzt eine einwandsfreie Methodik, weshalb auch eine genauere Darstellung der Technik und des Versuchsergebnisses dringend wünschenswert gewesen wäre.

Auch die anderen Schlüsse, die Schönfeld zieht, verlieren durch die Einseitigkeit des Materials an Bedeutung, vor allem die viel umstrittene Aufstellung der Grenzwerte der Zellzahl des normalen und pathologischen Liquors, dann aber auch die Beobachtung, daß bei kurz hintereinander folgenden Punktionen eine Vermehrung der Zellund Eiweißwerte des Liquors auftreten kann. Vom Kammerwasser immunisierter Tiere ist bekannt, daß nach Kammerpunktion eine Wiederholung des Eingriffes eiweiß- und antikörperreicheres Kammerwasser zutage fördert (R. Salus): Daß für die Liquorverhältnisse die Bedingungen ähnliche sind, ist noch nicht bewiesen (Walter, Kafka), jedoch ist, wenn Schönfelds Befunde zu Recht bestehen, daran zu denken, daß dieses Phänomen nur vorkommt, wenn eine Infektion der Meningen vorliegt oder bei der bestehenden Erkrankung (z. B. der Gonorrhöe) die Meningen, auch wenn sie noch nicht affiziert sind, einen Locus minoris resistentiae darstellen.

1) Siehe Kafka, Taschenbuch der praktischen Untersuchungsmethoden usw. Berlin, Julius Springer 1917. 
Zusammenfassend müssen wir daher sagen, daß wir in Schönfelds Arbeit eine wertvolle Studie über die Liquorverhältnisse bei Gonorrhöe und Hautkrankheiten erblicken, daß wir aber die von dem Autor gezogenen Schlüsse, soweit sie eine Verallgemeineruug für die Verhältnisse des sicher normalen Liquors bedeuten, ablehnen müssen.

Eine ins einzelne gehende Besprechung der Schönfeldschen Publikation wird an anderem Orte erfolgen. 